\title{
SCIENTIFIC REPORTS

\section{OPEN Effects of anti-H. pylori triple therapy and a probiotic complex on intestinal microbiota in duodenal ulcer}

Received: 8 January 2019

Accepted: 19 August 2019

Published online: 06 September 2019

\author{
Lili Wu ${ }^{1,2}$, Zikai Wang ${ }^{1}$, Gang Sun ${ }^{1}$, Lihua Peng ${ }^{1}$, Zhongsheng Lu ${ }^{1}$, Bin Yan ${ }^{1}$, Kun Huang ${ }^{1,3}$ \& \\ Yunsheng Yang ${ }^{1}$
}

This study aimed to investigate the intestinal microbiota in duodenal ulcer (DU) patients, effects of proton pump inhibitors, clarithromycin and amoxicillin, PCA) for Helicobacter pylori $(H$. pylori) and Bacillus subtilis and Enterococcus faecium (BSEF) on intestinal microbiota. DU patients were randomly assigned to receive either PCA (group TT) or PCA plus BSEF(groupTP). The fecal microbiome was conducted using high throughput $16 \mathrm{~S}$ rDNA gene and internal transcribed spacer sequencings. The diversity and abundance of intestinal bacteria in the DU were significantly lower than health check control (HC) group. In the TT group, the abundance and diversity of both intestinal bacteria and fungi decreased after PCA treatment, compared with those before treatment, whereas in the TP group no obvious changes were observed. In the TT group at all the time points, both the intestinal bacteria and fungi were different from those in the HC group. However, in the TP group, at 10w the bacterial flora abundance was close to that in the $\mathrm{HC}$ group. The results indicate that anti- $H$. pylori treatment induced significant decrease in the diversity of intestinal microbiota, while the combined therapy supplemented with BSEF could protect and restore the intestinal microbiota.

Guidelines about $H$. pylori eradication have stated that $H$. pylori infection should be eradicated in duodenal ulcer (DU) patients by the standard triple therapy ${ }^{1}$. Of the DU patients who received standard anti-H. pylori treatment, $10 \%$ to $30 \%$ develop antibiotic-associated diarrhea (AAD) and stool changes ${ }^{2}$. The reasons may relate to intestinal microbiota disorders caused by the use of anti-bacterial drugs ${ }^{3,4}$. Recent studies have reported that eradication of $H$. pylori caused perturbation of the gut microbiome and may indirectly affect the health of human ${ }^{5}$. However, the intestinal microbiota in DU patients is unknown. The effect on intestinal fungi caused by anti- H. pylori therapy, as well as a combined therapy with probiotic treatment in DU patients remains unclear. We hypothesized that (1) the intestinal microbiota in DU is different from that in healthy people; (2) H. pylori standard treatment disrupts intestinal microbiota; and (3) combined Bacillus subtilis and Enterococcus faecium(BSEF) treatment may improve the rate of $H$. pylori eradication, reduce side effects, and protect intestinal microbiota.

\section{Methods}

Study population. Healthy adults aged 18-65 years old were first screened to assess study eligibility. The exclusion criteria for the study were diabetes, hyper- or hypothyroidism, prior gastric or bariatric surgery, prior documented treatment for $H$. pylori, antibiotic or probiotics use within 4 weeks of enrollment, use of steroids or other immunomodulating drugs within 4 weeks of enrollment, recent vaccination and Charlson weighed comorbidity index $<2$. Written informed consent was obtained from qualified volunteers prior to study participation. Authors had access to the study data and reviewed and approved the final manuscript. The study protocol was reviewed and approved by the Medical Ethics Committee at the Chinese PLA General Hospital (PLAGH) (date of first registration:1/4/2013,registration number:S2013-051-01) and the Chinese Clinical Trial Registry (registration number:ChiCTR-TRC-13003228) on 19April 2013. We confirm that all methods were performed in accordance with the relevant guideline.

\footnotetext{
${ }^{1}$ Department of Gastroenterology, First Medical Center, Chinese People's Liberation Army General Hospital, Beijing, China. ${ }^{2}$ Department of Gastroenterology, Second Medical Center, Chinese People's Liberation Army General Hospital, Beijing, China. ${ }^{3}$ Department of Gastroenterology, Civil Aviation General Hospital, Beijing, China. Correspondence and requests for materials should be addressed toY.Y. (email: sunny301ddc@126.com)
} 
Each participant was asked to fill in 2 side-effect questionnaires to record the side effects according to severity: mild, moderate or severe ${ }^{6,7}$. The patients were also asked to record the improvement of symptoms and the status of ulcer healing under endoscopy before and after treatment ${ }^{8}$.

Sample collection. The active DU patients were diagnosed in the Chinese PLA General Hospital (PLAGH) from 2013 to 2016. Urea breathing test (UBT) was performed in all patients. Biopsy specimens were taken from the antrum and corpus for a rapid urease test (RUT) and histology. H. pylori was considered present if two of the three were positive. When taking a gastroscope, take $1-2 \mathrm{ml}$ of gastric juice, immerse the Acilit ${ }^{\circledR} \mathrm{pH}$ test strip into the measured gastric juice, and read the $\mathrm{pH}$ of the gastric juice.

After the patients met the eligibility criteria and provided informed consent, we subsequently randomized patients using concealed allocation based on a list of random numbers, which was computer-generated. Forty $H$. pylori infected patients with active DU were randomly divided into a triple treatment group $(\mathrm{TT}, \mathrm{n}=20)$ and a triple therapy plus probiotic treatment group (TP, $n=20$ ). In addition, 20 normal healthy persons were included as health check controls ( $\mathrm{HC}, \mathrm{n}=20$ ). Healthy persons meas no digestive diseases and other systemic diseases, and have not taken any drugs in the last month. Patients in the TT group received 2 weeks of standard triple therapy (20 mg esomeprazole, $500 \mathrm{mg}$ clarithromycin, $1000 \mathrm{mg}$ amoxicillin). Patients in the TP group received PCA followed by 6 weeks of the probiotics Bacillus Subtilis and coated capsules BSEF $500 \mathrm{mg} 3$ times daily. Follow-up endoscopy was conducted for all patients after stopping PCA for 4 weeks to check ulcer healing and $H$. pylori status. The volunteers were subsequently followed up at 2,4 , and 8 weeks post- $H$. pylori eradication. Stool samples (group TT fecal samples, TF; group TP fecal samples, PF) were collected pre-therapy and during each visit, and were frozen immediately at $-80^{\circ} \mathrm{C}$ until DNA extraction. TF1, TF2 and TF3 represent fecal samples of TT group for 2, 4 and 6 weeks of anti-Hp treatment, respectively. PF1, PF2 and PF3 represent fecal samples of TP group for 2, 4 and 6 weeks of anti-Hp treatment, respectively.

Gene amplification and sequencing. The gut microbial genomic DNA was extracted from stool samples using a stool DNA extraction kit (QIAamp DNA Stool Mini Kit; cat. no. 51504). The primers of the bacterial 16S rDNA were as follows: The upstream primer was 5'-GTGCCAGCMGCCGCGGTAA-3', and the downstream primer was 5'-GGACTACHVGGGTWTCTAAT-3'. The V4 region of stool bacterial $16 \mathrm{~S}$ rDNA and the ITS region of fungi were amplified with a high-fidelity enzyme. The ITS1 region primer is ITS1-5F-ITS2; the ITS2 region primer is: ITS2-3F-ITS2-4R. The library was constructed using New England Biolabs' NEB Next ${ }^{\circledR}$ UltraTM DNA Library Prep Kit for Illumina library. Sequencing was completed using a Paired-End (PE) approach on the Illumina MiSeq high-throughput sequencing platform.

Bioinformatics analysis. Quality control of the reads was performed by the QIIME software package to pick the high-quality reads that met the requirement ${ }^{9,10}$. Sequences with similarity greater than $97 \%$ were picked up using the operation command of the QIIME quality controller and were clustered into an operational taxonomic unit (OTU) ${ }^{11}$. The Ribosomal Database Project (RDP) software package was used for the homology alignment, as well as the species and genera taxonomic identification of the longest $16 \mathrm{~S}$ rDNA sequence fragment of OTUs $^{12}$. The database referred to the Greenbank at http://greengenes.lbl.gov/cgi-bin/nph-index.cgi. The resulting UniFrac distance matrices were used to perform Principal Coordinate Analysis (PCoA) to determine the similarity between groups of samples/time-points.

Statistical methods. The raw data of the taxonomy summary results was exported to SPSS software version 20.0 (SPSS Inc., Chicago, IL) for statistical analysis. The mean abundance in percentage (\%) and the $95 \%$ confidence interval (95\% CI) for the phyla of stool microbiome at different time-points were calculated. Parametric paired-samples t-test was performed to compare the genera of the stool microbiome. Pearson's Correlation Coefficient was also calculated to investigate across different time points; a two-tailed $P$-value of $<0.05$ was considered significant.

Compliance with ethical standards. The study protocol was reviewed and approved by the Medical Ethics Committee at the Chinese PLA General Hospital (PLAGH) (date of first registration:1/4/2013,registration numberS2013-051-01) and the Chinese Clinical Trial Registry (date of first registration:19/4/2013, registration number:ChiCTR-TRC-13003228).

\section{Results}

Clinical information. There was no significant difference in gender, age, and BMI among the 3 groups $(P<0.05)$, suggesting the comparability of the groups (Table 1$)$.

H. pylori eradication rate. Gastroscopy revealed that the ulcer healing rate of the TT group (90\%) was lower than that of the TP group $(100 \%)$, but there was no statistically significant difference $(P=0.4682)$.

A total of 16 cases (80\%) in the TT group were successfully eradicated, and 17 cases (85\%) were successfully eradicated in the TP group. No difference in the success rate of the H. pylori eradication was observed between the 2 groups $(P>0.05)$.

Gastric $\mathrm{pH}$ value. The gastric $\mathrm{pH}$ values of DU patients in the pre-TT and the pre-TP groups were significantly different from that in the HC group $(P<0.01)$, while the TT and the TP groups after treatment (post-TT and post-TP groups) showed no difference with the HC group $(P=0.274)$. Significant differences were observed between the pre-TT and the post-TT group $(P<0.01)$, as well as between the pre-TP and post-TP groups $(P<0.01)$ (Table 2 and Fig. 1$)$. 


\begin{tabular}{|l|l|l|l|l|}
\hline Basic information & TT group & TP group & HC group & P-value \\
\hline Number of cases & 20 & 20 & 20 & \\
\hline Gender & 13 & 12 & 12 & 0.9319 \\
\hline Male & 7 & 8 & 8 & \\
\hline Female & $45.5 \pm 8.4$ & $45.2 \pm 11.6$ & $42.9 \pm 12.3$ & 0.2386 \\
\hline Ages & $24.1 \pm 2.6$ & $24.4 \pm 2.7$ & $24.5 \pm 3.0$ & 0.8112 \\
\hline BMI
\end{tabular}

Table 1. Basic information of the subjects.

\begin{tabular}{|l|l|l|l|l|}
\hline Group & $\begin{array}{l}\text { Number } \\
\text { of cases }\end{array}$ & $\begin{array}{l}\text { pH value Before } \\
\text { treatment }\end{array}$ & $\begin{array}{l}\text { pH value After } \\
\text { treatment }\end{array}$ & P-value \\
\hline TT Group & 20 & $0.720 \pm 0.330$ & $1.395 \pm 0.613$ & $0.0001^{*}$ \\
\hline TP Group & 20 & $0.720 \pm 0.321$ & $1.555 \pm 0.610$ & $0.0000^{*}$ \\
\hline HC Group & 20 & $1.265 \pm 0.456$ & & \\
\hline
\end{tabular}

Table 2. $\mathrm{pH}$ value of gastric juice before and at $4 \mathrm{w}$ after treatment (Mean $\pm \mathrm{SD})$.



Figure 1. Gastric $\mathrm{pH}$ value before and after treatment.

Side effects. There were more side effects in the TT group than in the TP group (35\% vs $20 \%$ ). The most common side effects were diarrhea (35\%), followed by nausea (30\%), abdominal pain (25\%), abnormal taste (20\%), abdominal bloating (15\%), anorexia (10\%), and constipation (5\%). These side effects mostly occurred during treatment, and were predominantly mild to moderate, whereas all the cases of the TP group were mild.

Fecal microbiome in DU patients. The average Raw PE number obtained for each bacterial sample was 65630 and fungi sample was 54549. The dilution curve was drawn by 3 methods, including the OTU numbers, the Chaol and the Shannon 3 for mutual validation, and the results suggested a trend of reaching gentleness at 10,000 sequences.

Bacterial microbiome. OTU analysis: Statistical analysis showed that the OTU number was lower in the DU group compared with that in the HC group, either at the 97\% OTU species-level (HC group $505.33 \pm 133.63$ vs DU group $355.07 \pm 104.11 P=0.0003$ ) or at the 95\% OTU genus level (HC group $412.17 \pm 114.75$ vs DU group $271.28 \pm 89.73, P=0.0001$ ). Differences between the 2 groups were statistically significant (Fig. 2 ).

Annotation of species and analysis of differential bacteria: The statistical analysis revealed that the bacterial flora community was derived from 38 bacterial phyla, 110 bacterial classes, 157 bacterial orders, 230 bacterial families, and 275 bacterial genera.

At the phylum level, the abundance distribution showed that in the HC group, the dominant phyla were Firmicutes, Bacteroidetes, and Proteobacteria. These 3 bacterial phyla were abundant in every sample with high percentages, accounting for $45.5 \%, 33.0 \%$, and $13.2 \%$, respectively, and together accounting for $91.7 \%$ of the total bacteria. In the DU group, the dominant phyla were also Firmicutes, Bacteroidetes, and Proteobacteria, accounting for $52.5 \%, 25.7 \%$, and $15.7 \%$, respectively, and together accounting for $93.9 \%$ of the total bacteria. Although the percentages of the 3 bacterial phyla have changed, the differences between the DU group and the HC group were not statistically different. Comparing the other 35 bacterial phyla between the DU group and the HC group revealed a total of 7 bacterial phyla with statistically significant differences: Actinobacteria, Gemmatimonadetes, Nitrospirae, Chlorobi, Thermi, WS3, and Caldithrix. The percentages of these 7 bacterial phyla were all lower in the DU group compared with those in the HC group. 




Figure 2. Comparison of OTUs from the 2 groups at the species and genus levels.

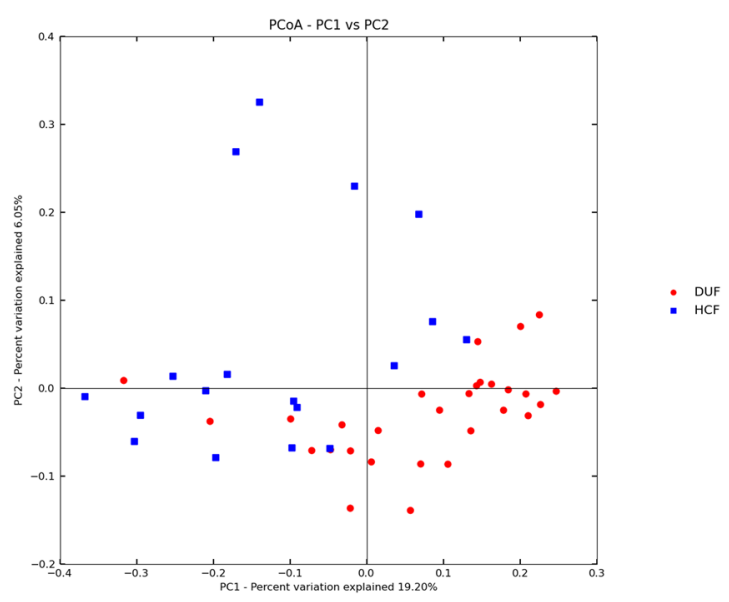

Figure 3. PCoA analysis (blue denotes the HC group and red denotes the DU group).

At the genus level, the dominant genera in the HC group were Bacteroides (22.8\%), Faecalibacterium (13.6\%), Prevotella (5.3\%), Roseburia (5.1\%), Ruminococcus (4.5\%), Escherichia (4.2\%), Bifidobacterium (4.2\%), Lachnospira (2.3\%), etc.; the dominant genera in the DU group were Bacteroides (17.1\%), Faecalibacterium (15.3\%), Escherichia (7.8\%), Ruminococcus (5.5\%), Roseburia (4.7\%), Prevotella (4.6\%), Akkermansia (2.9\%), Parabacteroides (2.4\%), Bifidobacterium (2.0\%), etc. There were a total of 55 differential bacteria at the genus level, 54 of which were less in the DU group than those in the HC group, except the Natronomonas genus that increased in the DU group.

Analysis of species composition: The PCoA analysis found that the intra-group distances between each sample within the HC group and the DU group were smaller than the inter-group distances, suggesting the similarity of the species compositions (Fig. 3).

The UPGMA (Unweighted Pair Group Method with Arithmetic Mean) method was used to input the unweighted UniFrac clustering matrix, which performed cluster analysis on samples. In the cluster diagram (Fig. 4), we found that the bacterial flora structure among all the samples exhibited both clustering and various degrees of overlapping. However, only a few samples could be clustered between the DU group and the HC group, with a general trend of high similarity between samples within groups. Therefore, the DU group or the HC group was considered as a homologous group, and samples from such groups exhibited internal clustering.

Fungi microbiome. Fungi microbiome. OTU analysis: The OTU number was lower in the DU group compared with that in the HC group, either at the 97\% OTU species level (HC group 60.70 \pm 14.46 vs DU group $48.65 \pm 10.26 P=0.0180$ ) or at the $95 \%$ OTU genus level (HC group $51.20 \pm 15.49$ vs DU group $38.77 \pm 9.13 P=0.0141)$. Differences between the 2 groups were statistically significant (Fig. 5 ).

Annotation of species and analysis of differential fungi: According to the annotation results of species, most of the fungal communities came from 2 fungal phyla, 31 fungal classes, 53 fungal orders, 90 fungal families, and 104 fungal genera.

At the phylum level, the abundance distribution showed that the abundance of Ascomycota was 59.6\% in the HC group and $58.8 \%$ in the DU group; the abundance of Basidiomycota was $37.0 \%$ in the HC group and $40.3 \%$ in the DU group. There was no difference in the fungal phyla between the 2 groups.

At the genus level, the abundance distribution showed that in the HC group the dominant genera with the fungal flora abundance of more than $1 \%$ were Sarcinomyces (49.2\%), Malassezia (33.1\%), Alternaria (5.9\%), Pseudozyma (2.4\%), Simplicillium (1.9\%), and Aspergillus (1.5\%); in the DU group, the dominant genera with the fungal flora abundance of more than $1 \%$ were Sarcinomyces (53.9\%), Malassezia (35.8\%), Pseudozyma (3.6\%), Simplicillium (2.2\%), and Aspergillus (1.0\%). There were 2 differential fungi at the genus level: Pseudozyma 

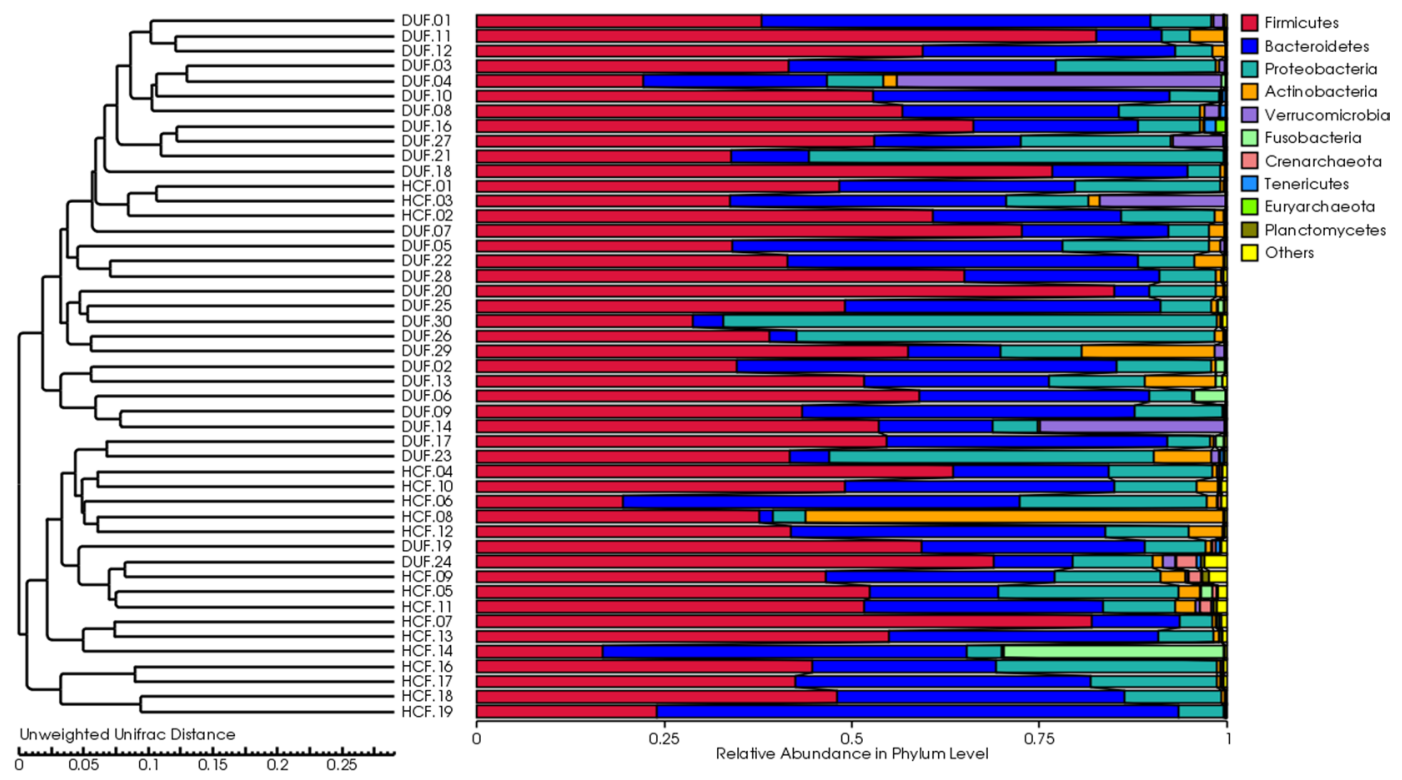

Figure 4. Unweighted UniFra distance clustering tree of samples.

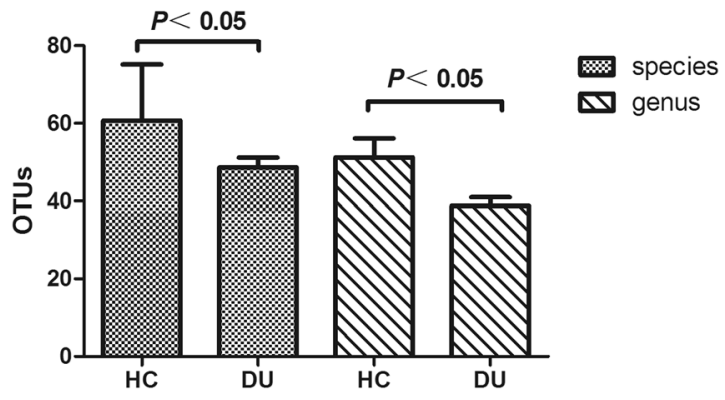

Figure 5. Comparison of OTUs of the 2 groups of at the species and genus level.

significantly increased in DU (3.6\%) compared with that in HC (2.4\%) $(P=0.01499)$, and Candida significantly decreased in DU $(0.001 \%)$ compared with that in $\mathrm{HC}(0.024 \%)(P=0.02113)$.

Analysis of species composition: PCoA analysis was performed, and the results are shown in Fig. 6. It can be seen that the majority of the samples from the DU groups were clustered in the lower left quadrant, whereas samples from the HC group tended to locate in the upper part of the quadrant. These results suggest that the fungal flora compositions between the DU group and the HC group are not exactly the same, while the internal fungal flora structures within groups are more similar.

Next, samples were subjected to cluster analysis (Fig. 7). In the clustering diagram, we found that the fungal flora structure among all the samples exhibited both clustering and various degrees of overlapping. However, only a few samples could be clustered between the DU group and the HC group. The general trend was that samples from the DU group or the HC group were first clustered, suggesting that either the DU group or the HC group had a high intra-group similarity.

Changes of intestinal stool microbiome after anti-H. pylori treatment. Bacterial microbiome. OTU analysis: Statistical analysis of OTUs at the bacterial species level was carried out for 4 different time points in the TT group and the TP group (Table 3 and Fig. 8); 0 week (w) represents the DU group before treatment (i.e., with ulcers). Intra-group comparisons were first performed within both groups, and we found that, except in the TT group where differences were observed between DU and TF1 $(P=0.03159)$ and between DU and TF2 $(P=0.04526)$, there was no statistical difference in the rest of the pairwise comparisons. In other words, at the end of the anti-H. pylori treatment and at $6 \mathrm{w}$ after treatment, the intestinal bacterial flora abundance in the TT group decreased compared with that in the untreated group, whereas the TP group showed no significant changes compared with the untreated group.

When the 2 groups were compared with the HC group before and after treatment at different time points, respectively, most of the compared groups showed statistical differences, except between the TP group and the HC group (Table 4).

Annotation of species and analysis of differential bacteria: 


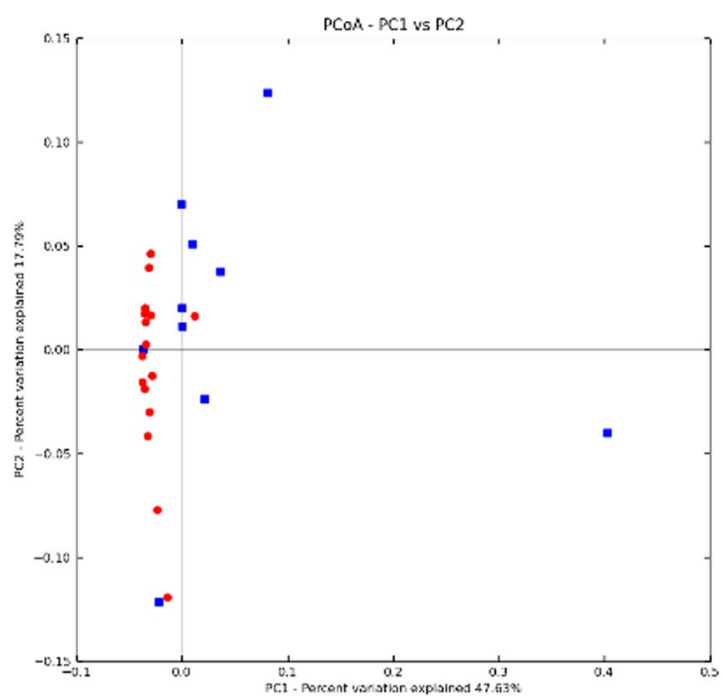

Figure 6. PCoA analysis (blue represents the HC group, and red represents the DU group).



Figure 7. Clustering tree.

\begin{tabular}{|l|l|l|}
\hline & TT Group & TP Group \\
\hline 0w & $347.41 \pm 82.61$ & $335.66 \pm 85.43$ \\
\hline $2 \mathrm{w}$ & $302.40 \pm 48.11^{*}$ & $342.67 \pm 68.58 \#$ \\
\hline $6 \mathrm{w}$ & $310.07 \pm 34.14^{*}$ & $346.07 \pm 63.33 \#$ \\
\hline $10 \mathrm{w}$ & $316.40 \pm 48.02$ & $400.00 \pm 141.34 \#$ \\
\hline
\end{tabular}

Table 3. OTUs at different time points in the two groups. (*denotes $P<0.05$ compared with $0 \mathrm{w}$, and \# denotes $P<0.05$ when comparing the 2 groups at the same time points).

(1) The intestinal bacterial changes between groups at the same time points. At the end of the $2 \mathrm{w}$ treatment, there was no significant change in intestinal bacterial flora at the phylum level between the 2 groups, whereas at the genus level, a total of 23 differential bacterial genera $(P<0.05)$ were discovered. After $6 \mathrm{w}$ of treatment, there were 28 differential bacterial genera, and at $10 \mathrm{w}$, there were 11 . Details of the differential bacterial genera are listed in the following table (Table 5).

(2) The intestinal bacterial changes within groups at different time points. In the TT group, among the top ten bacterial phyla at the phylum level, pairwise comparisons within the group showed that differences were only observed in the Bacteroidetes phylum and the Tenericutes phylum: the relative abundance of the Bacteroidetes phylum increased in TF2 compared with that in DU $(P=0.03664)$, and the relative abundance of the Tenericutes phylum decreased in TF1 compared with that in DU $(P=0.01807)$. There was no statistical difference in changes of different bacterial phyla at the remaining time points (Fig. 9). 


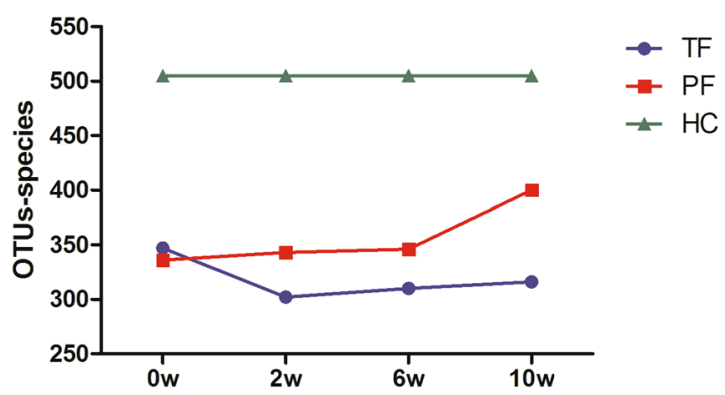

Figure 8. OTUs at different time points before and after treatment.

\begin{tabular}{|l|l|l|l|l|}
\hline Group & HC & PF1 & PF2 & PF3 \\
\hline HC & - & $0.00068 * *$ & $0.00043 * *$ & 0.11850 \\
\hline TF1 & $0.00000 * *$ & $0.02077 *$ & - & - \\
\hline TF2 & $0.00000 * *$ & - & $0.01052 *$ & - \\
\hline TF3 & $0.00001 * *$ & - & - & $0.03916^{*}$ \\
\hline
\end{tabular}

Table 4. $P$-values from the inter-group comparisons of the OTUs at different time points. $(*$ denotes $P<0.05$, $* *$ denotes $P<0.01)$.

\begin{tabular}{|c|c|c|c|}
\hline & $2 w$ & $6 w$ & $10 w$ \\
\hline \multirow{26}{*}{$\begin{array}{l}\text { The bacterial genera higher in the } \\
\text { TP group than the TT group }\end{array}$} & Actinomadura & Actinomadura & Achromobacter \\
\hline & Atopobium & Anaerofilum & Actinomyces \\
\hline & Brevundimonas & Candidatus Nitrososphaera & Bradyrhizobium \\
\hline & Butyrivibrio & Candidatus Solibacter & Candidatus Solibacter \\
\hline & Coprococcus & Coprococcus & Coprococcus \\
\hline & Coraliomargarita & Coraliomargarita & Chelativorans \\
\hline & Corynebacterium & Dechloromonas & Cetobacterium \\
\hline & Desulfobacca & Desulfomonile & Cupriavidus \\
\hline & Desulfobulbus & Desulfobulbus & \\
\hline & Eggerthella & Dok59 & \\
\hline & Faecalibacterium & Dorea & Thiobacillus \\
\hline & GOUTA19 & Leuconostoc & Leuconostoc \\
\hline & Helicobacter & Luteimonas & \\
\hline & Lewinella & Lewinella & \\
\hline & & Luteolibacter & \\
\hline & & Neisseria & \\
\hline & & Nitrosopumilus & \\
\hline & Oscillospira & Oscillospira & \\
\hline & Rhodobacter & Parapedobacter & \\
\hline & Rhodoplanes & Planctomyces & \\
\hline & Roseburia & Thauera & \\
\hline & Roseomonas & Syntrophobacter & \\
\hline & Rubrivivax & Syntrophomonas & \\
\hline & Thauera & $T 78$ & \\
\hline & & Thermomonas & \\
\hline & Thiobacillus & Thiobacillus & \\
\hline \multirow{2}{*}{$\begin{array}{l}\text { The bacterial genera lower in the } \\
\text { TP group than the TT group }\end{array}$} & Dialister & Dialister & Dialister \\
\hline & & Plesiomonas & \\
\hline
\end{tabular}

Table 5. Differential bacterial genera between the 2 groups at different time points.

In the TP group, among the top 10 bacterial phyla at phylum level, pairwise comparisons within the group showed that differences were only observed in the Firmicutes phylum, the relative abundance of which increased in PF2 compared with that in PF1 $(P=0.01356)$. There was no statistical difference in changes of different bacterial phyla at the remaining time points (Fig. 10). 


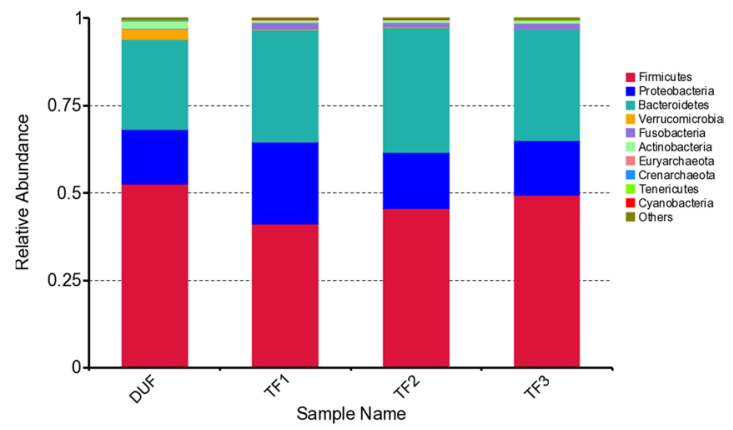

Figure 9. Abundance changes of bacterial phyla in the TT group before and after treatment.



Figure 10. Abundance changes of bacterial phyla in the TP group before and after treatment.

At the genus level, among the 4 different time points in the TT group, pairwise comparisons within the group revealed a total of 8 significantly different $(P<0.05)$ bacterial genera, the bacterial flora abundance of which was more than $0.1 \%$ of the total samples.

At the genus level, among the 4 different time points in the TP group, pairwise comparisons within the group revealed a total of 5 significantly different $(P<0.05)$ bacterial genera, the bacterial flora abundance of which was more than $0.1 \%$ of the total samples. Such differential bacterial genera were fewer in the TP group than that in the TT group, and their abundance percentage was very low. These results suggest that the changes of bacteria at different time points in the TP group were slight, and were from only a small number of bacteria. These bacterial changes were stable, and exhibited a trend of gradual transition. In the TT group, however, the bacterial changes were larger, came from a larger number of bacteria, and presented more intense amplitudes.

Fungi microbiome. OTU analysis: The results revealed that at the 3 time points after TT treatment, the number of OTUs of the intestinal fungal flora decreased compared with that in DU, and was statistically different $(P<0.05)$; before and after TP treatment, however, there was no obvious change in the number of OTUs. There were differences between the 2 treatment approaches at the same time points (Table 6 and Fig. 11).

When the 2 groups were compared with the HC group before and after treatment at different time points, respectively (Table 7), it could be seen that there was no statistical difference among the 3 time points after TP treatment and the HC group, whereas there were statistically significant differences among the 3 time points in the TT group and the HC group.

Annotation of species and analysis of differential fungi:

(1) The intestinal fungal changes between groups at the same time points. At the phylum level, the 2 treatment approaches showed no obvious difference in the intestinal fungal flora at any of the 3 time points after treatment. At the genus level, some of the fungi were significantly different $(P<0.05)$ : at the end of $2 \mathrm{w}$ treatment, a total of 5 differential fungal genera were observed. Details of the differential fungal genera are listed in the following table (Table 8).

(2) Intestinal fungal changes within groups at different time points At the phylum level, there was no difference among the 4 time points either in the TP group or in the TT group. At the genus level, among the 4 time points in the TT group, pairwise comparisons within the group revealed that only the Pseudozyma genus and the Cladosporium genus showed significant differences $(P<0.05)$, and the trends of the changes at the 4 time points are shown in the figure below; among the 4 time points in the TP group, pairwise comparisons within the group revealed a total of 6 differential fungal genera $(P<0.05)$, more than those in the TT group. Moreover, the relative abundance of the differential fungal genera was higher than that in the TT group, suggesting that the fungal changes at different time points in the TT group were slight and came from only a small number of fungi, whereas the fungal changes in the TP group were larger and came from a larger number of fungi. 


\begin{tabular}{|l|l|l|}
\hline & TT Group & TP Group \\
\hline $0 \mathrm{w}$ & $46.12 \pm 9.44$ & $51.00 \pm 10.38$ \\
\hline $2 \mathrm{w}$ & $37.71 \pm 8.03^{*}$ & $55.62 \pm 6.69 \#$ \\
\hline $6 \mathrm{w}$ & $35.30 \pm 5.50^{*}$ & $58.14 \pm 6.20 \#$ \\
\hline $10 \mathrm{w}$ & $37.62 \pm 6.02 *$ & $55.25 \pm 10.27 \#$ \\
\hline
\end{tabular}

Table 6. OTUs at different time points in the two groups. (*denotes $P<0.05$ compared with $0 \mathrm{w}$, and \# denotes $P<0.05$ when comparing the 2 groups at the same time points).

\begin{tabular}{|l|l|l|l|l|}
\hline Group & HC & PF1 & PF2 & PF3 \\
\hline HC & & 0.28750 & 0.40350 & 0.2505 \\
\hline TF1 & $0.00442 * *$ & $0.00021 * *$ & & \\
\hline TF2 & $0.00060 * *$ & & $0.00043 * *$ & \\
\hline TF3 & $0.00318 * *$ & & & $0.01979 *$ \\
\hline
\end{tabular}

Table 7. $P$ values from the inter-group comparisons of the OTUs at different time points. $(*$ denotes $P<0.05$, ** denotes $P<0.01)$.

\begin{tabular}{|l|l|l|l|}
\hline & $\mathbf{2 w}$ & $\mathbf{6 w}$ & $\mathbf{1 0 w}$ \\
\hline $\begin{array}{l}\text { The fungal genera higher in the TP } \\
\text { group than the TT group }\end{array}$ & Aspergillus & Trichoderma & Aureobasidium \\
\hline \multirow{4}{*}{$\begin{array}{l}\text { The fungal genera lower in the TP } \\
\text { group than the TT group }\end{array}$} & Cladosporium & Cladosporium & Cladosporium \\
\cline { 2 - 4 } & Pseudozyma & Pseudozyma & Pseudozyma \\
\cline { 2 - 4 } & Simplicillium & Simplicillium & Simplicillium \\
\cline { 2 - 4 } & & Sarcinomyces & Sarcinomyces \\
\cline { 2 - 4 } & Fusarium & & Fusarium \\
\cline { 2 - 4 } & & & Candida \\
\hline
\end{tabular}

Table 8. Differential fungal genera between the two groups at different time points.

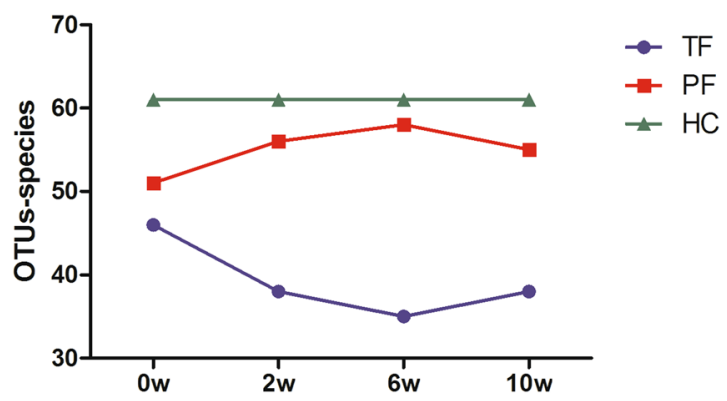

Figure 11. OTUs at different time points before and after treatment.

\section{Discussion}

Eradication of $H$. pylori is essential for the treatment of the H. pylori-associated DU². PCA therapy for $H$. pylori eradication has been in clinical use for a long time. Due to the increased rates of drug resistance and side effects, most studies have shown that the addition of probiotics reduces side effects, but whether these probiotics additives improve the eradication rate is still controversial ${ }^{6,13-16}$. Chen et al. ${ }^{17}$ found that Lactobacillus delbrueckii and Lactobacillus plantarum-18 additives had significant anti- $H$. pylori effects. The anti- $H$. pylori effects of the 2 strains mainly related to the $\mathrm{pH}$ value of the cells' supernatant and their metabolites.

However, some researchers have questioned the methods that combine anti-H. pylori therapy with probiotics additives ${ }^{18}$. But the efficacy of the therapy can be increased if acid-resistant and bile salts-resistant BSEF preparations are added. Park et al. ${ }^{19}$ have shown that the therapy combining 2 live bacteria-BSEF improved eradication rates and reduced side effects. Results from our study showed that the combination therapy with BSEF did not significantly improve eradication rates but reduced the incidence of side effects.

The application of PPIs in triple therapy increased the $\mathrm{pH}$ value of gastric juice, resulting in a low acidic environment of the stomach. The low acidic environment weakens the bacteria killing effect of the therapy, and may change intestinal microecology ${ }^{20}$. Our study found that the $\mathrm{pH}$ value of the gastric juice in DU patients $(0.72)$ was significantly lower than that in normal people (1.27). The $\mathrm{pH}$ value returned to normal after anti- $H$. pylori 
treatment. We have investigated, for the first time, the intestinal bacterial and fungal flora in DU, and found that the diversity and abundance of intestinal bacteria and fungi were significantly lower than those in healthy people.

The presence of human intestinal microbiota is vital for the health of the host. These microorganisms play a unique role in human nutrition, growth, metabolism, resistance to pathogens, immune regulation, and the like ${ }^{21-23}$. However, the use of antibiotics may break the steady state of the interaction between the microorganisms and the host ${ }^{24,25}$. Meanwhile, the impact of PPIs on intestinal microbiota has also been of concern ${ }^{26,27}$. PPIs weaken the role of gastric acid in eliminating exogenous bacteria, leading to easy invasion and colonization of exogenous pathogenic bacteria of the human body. A number of studies have confirmed that when receiving the combined application of PPIs and antibiotics for $H$. pylori infection, $10 \%$ to $30 \%$ of the patients experienced changes in defecation frequency and stool characteristics ${ }^{28-32}$. The reasons for this may relate to intestinal microbiota disturbance. This was also confirmed in our study, which has revealed that the incidence of diarrhea was as high as $35 \%$ during triple therapy.

Oh et al..$^{28}$ showed that probiotic supplementation can reduce the antibiotic-induced alteration and imbalance of the intestinal microbiota composition. Despite the changes in intestinal microbiota, this study suggested that the impact of triple therapy on the intestinal microbiota was short-term about 1 month. Our study found that anti-H. pylori treatment led to an imbalance of intestinal homeostasis and the reduction of diversity, especially during the triple only treatment. After combination therapy with probiotics additives, changes of intestinal microbiota were more moderate: intestinal bacterial diversity at the $10^{\text {th }}$ week was not significantly different from that in healthy people, while intestinal fungal diversity has always remained similar to that in the HC group.

Studies based on traditional microscopy have revealed a high incidence of fungal infection in patients with gastric ulcer, gastric cancer and gastric remnants ${ }^{33,34}$. So far, there is no report on sequencing results of intestinal microbiota in DU patients. Our study applied a high-throughput sequencing method, for the first time, to sequence intestinal fungal colonies in DU patients. The annotation of species found that the fungal communities were from 2 fungal phyla, 31 fungal classes, 53 fungal orders, 90 fungal families, and 104 fungal genera. In the HC group, the dominant genera were different with the DU group. The probiotic drugs used in our study contain 2 important strains of bacteria that promote the health of human intestines. In addition to the contributions from the bacteria themselves, these strains also promote the growth of other bacteria, especially thick-walled bacteria, such as Bifidobacterium. In the probiotic preparation we used, E. faecium is a gram-positive and facultative anaerobic bacterium. This bacterium reproduces rapidly, and has a strong inhibitory effect on pathogenic bacteria; $B$. subtilis is a gram-positive and aerobic bacterium, which consumes intestinal oxygen upon its intestinal colonization, resulting in biological oxygen deprivation. Such biological oxygen deprivation leads to a reduction in local oxygen concentration, creating an appropriate environment for the growth of normal intestinal anaerobic microbiota. This promotes the growth of Bifidobacterium, Lactobacillus, enterococci, and other beneficial bacteria, and inhibits the growth of Escherichia coli, dysentery bacili, Staphylococcus aureus, and other pathogenic bacteria, thereby restoring the balance of intestinal microbiota ${ }^{33}$. Studies have also found that B. subtilis produces a variety of digestive enzymes, further breaking down protein, fat, carbohydrates, and fibrin, etc. B. subtilis also produces lysozyme and more than 80 types of antibacterial compounds, thereby inhibiting the colonization and growth of pathogenic bacteria ${ }^{34}$. Moreover, B. subtilis inhibits the activity of all the $H$. pylori microbeads by secreting amicoumacin A and non-amicoumacin antibiotics. This inhibitory effect is not subject to acid restriction and is resistant to proteolytic cleavage. Based on the aforementioned mechanisms, BSEF formulations can be used against the intestinal microbiota imbalance caused by triple therapy.

\section{Data Availability}

The datasets generated and/or analysed during the current study are available from the corresponding author and first author on resonable request.

\section{References}

1. Malfertheiner, P. et al. Management of Helicobacter pylori infection-the Maastricht IV/ Florence Consensus Report. Gut 61, 646-664 (2012).

2. Asaka, M. et al. Follow-up survey of a large-scale multicenter, double-blind study of triple therapy with lansoprazole, amoxicillin, and clarithromycin for eradication of Helicobacter pylori in Japanese peptic ulcer patients. J. Gastroenterol. 38, 339-347 (2003).

3. Vesper, B. J. et al. The effect of proton pump inhibitors on the human microbiota. Curr Drug Metab 10, 84-89 (2009).

4. Bartlett, J. G. Clinical practice. Antibiotic-associated diarrhea. N Engl J Med 346, 334-339 (2002).

5. Yap, T. W. et al. Helicobacter pylori eradication causes perturbation of the human gut microbiome in young adults. PLoS One 11, e0151893 (2016).

6. Cindoruk, M., Erkan, G., Karakan, T., Dursun, A. \& Unal, S. Efficacy and safety of Saccharomyces boulardii in the 14-day triple antiHelicobacter pylori therapy: a prospective randomized placebo-controlled double-blind study. Helicobacter 12, 309-316 (2007).

7. Armuzzi, A. et al. The effect of oral administration of Lactobacillus GG on antibiotic-associated gastrointestinal side-effects during Helicobacter pylori eradication therapy. Aliment Pharmacol Ther 15, 163-169 (2001).

8. De Luca, L. et al. Measuring dyspepsia: a new severity index validated in Bologna. Dig Liver Dis 36, 806-810 (2004)

9. Wang, Q., Garrity, G. M., Tiedje, J. M. \& Cole, J. R. Naive Bayesian classifier for rapid assignment of rRNA sequences into the new bacterial taxonomy. Appl Environ Microbiol 73, 5261-5267 (2007).

10. Caporaso, J. G. et al. Global patterns of $16 \mathrm{~S}$ rRNA diversity at a depth of millions of sequences per sample. Proc Natl Acad Sci USA 108(Suppl 1), 4516-4522 (2011)

11. Edgar, R. C. UPARSE: highly accurate OTU sequences from microbial amplicon reads. Nat Methods 10, 996-998 (2013).

12. Caporaso, J. G. et al. QIIME allows analysis of high-throughput community sequencing data. Nat Methods 7, 335-336 (2010).

13. Zhou, L. et al. A comparative study of sequential therapy and standard riple therapy for Helicobacter pylori Infection: A Randomized Multicenter Trial. Am J Gastroenterol 109, 535-541 (2014).

14. Duman, D. G. et al. Efficacy and safety of Saccharomyces boulardii in prevention of antibiotic-associated diarrhoea due to Helicobacter pylori eradication. Eur J Gastroenterol Hepatol 17, 1357-1361 (2005).

15. Wang, K. Y. et al. Effects of ingesting Lactobacillus- and Bifidobacterium-containing yogurt in subjects with colonized Helicobacter pylori. Am J Clin Nutr 80, 737-741 (2004). 
16. Kim, M. N. et al. The effects of probiotics on PPI-triple therapy for Helicobacter pylori eradication. Helicobacter 13, 261-268 (2008).

17. Chen, X. et al. Antagonistic activities of lactobacilli against Helicobacter pylori growth and infection in human gastric epithelial cells. J Food Sci 77, M9-14 (2012).

18. Dai, C., Zheng, C. Q. \& Jiang, M. Efficacy of probiotics in Helicobacter pylori eradication therapy. J Clin Gastroenterol 47, 814-815 (2013).

19. Park, S. K. et al. The effect of probiotics on Helicobacter pylori eradication. Hepatogastroenterology 54, 2032-2036 (2007).

20. Guerre, J., Vedel, G., Gaudric, M., Paul, G. \& Cornuau, J. Bacterial flora in gastric juice taken at endoscopy in 93 normal subjects. Pathol Biol (Paris) 34, 57-60 (1986).

21. Perez-Cobas, A. E. et al. Gut microbiota disturbance during antibiotic therapy: a multi-omic approach. Gut 62, 1591-1601 (2013).

22. Lynch, S. V. \& Pedersen, O. The human intestinal microbiome in health and disease. N Engl J Med 375, 2369-2379 (2016).

23. Kahrstrom, C. T., Pariente, N. \& Weiss, U. Intestinal microbiota in health and disease. Nature 535, 47 (2016).

24. Ianiro, G., Tilg, H. \& Gasbarrini, A. Antibiotics as deep modulators of gut microbiota: between good and evil. Gut 16, 312297 (2016).

25. Blaser, M. J. Antibiotic use and its consequences for the normal microbiome. Science 352, 544-545 (2016).

26. Jackson, M. A. et al. Proton pump inhibitors alter the composition of the gut microbiota. Gut 65, 749-756 (2016)

27. Imhann, F. et al. Proton pump inhibitors affect the gut microbiome. Gut 65, 740-748 (2016).

28. Oh, B. et al. The effect of probiotics on gut microbiota during the Helicobacter pylori eradication: Randomized Controlled Trial. Helicobacter 21, 165-174 (2016).

29. Buhling, A., Radun, D., Muller, W. A. \& Malfertheiner, P. Influence of anti-Helicobacter triple-therapy with metronidazole, omeprazole and clarithromycin on intestinal microflora. Aliment Pharmacol Ther 15, 1445-1452 (2001).

30. Adamsson, I., Nord, C. E., Lundquist, P., Sjostedt, S. \& Edlund, C. Comparative effects of omeprazole, amoxycillin plus metronidazole versus omeprazole, clarithromycin plus metronidazole on the oral, gastric and intestinal microflora in Helicobacter pylori-infected patients. J. Antimicrob Chemother 44, 629-640 (1999).

31. Lou, J. G., Chen, J., Huang, X. L. \& Zhao, Z. Y. Changes in the intestinal microflora of children with Helicobacter pylori infection and after Helicobacter pylori eradication therapy. Chin Med J (Engl) 120, 929-931 (2007).

32. Sheu, B. S. et al. Impact of supplement with Lactobacillus- and Bifidobacterium-containing yogurt on triple therapy for Helicobacter pylori eradication. Aliment Pharmacol Ther 16, 1669-1675 (2002).

33. Tompkins, T. A., Hagen, K. E., Wallace, T. D. \& Fillion-Forte, V. Safety evaluation of two bacterial strains used in Asian probiotic products. Can J Microbiol 54, 391-400 (2008).

34. Pinchuk, I. V. et al. In vitro anti-Helicobacter pylori activity of the probiotic strain Bacillus subtilis 3 is due to secretion of antibiotics. Antimicrob Agents Chemother 45, 3156-3161 (2001).

\section{Acknowledgements}

This study was supported by the National High-Tech 863 Program of China (2015AA020701). The authors would like to thank this Program for its assistance and support. We would like to thank Chen Wei for her support in translation of paper.

\section{Author Contributions}

L.L.W., Z.K.W. and Y.S.Y. Study concept and design; Z.S.L. and B.Y. acquisition of samples; L.L.W., H.K. and Z.K.W. acquisition of data, analysis of data and interpretation of data; L.L.W. drafting of the manuscript; G.S., L.H.P. and Y.S.Y. critical revision of the manuscript for important intellectual content; Y.S.Y. obtained funding. All the authors contributed to manuscript revision.

\section{Additional Information}

Competing Interests: The authors declare no competing interests.

Publisher's note: Springer Nature remains neutral with regard to jurisdictional claims in published maps and institutional affiliations.

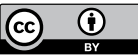

Open Access This article is licensed under a Creative Commons Attribution 4.0 International License, which permits use, sharing, adaptation, distribution and reproduction in any medium or format, as long as you give appropriate credit to the original author(s) and the source, provide a link to the Creative Commons license, and indicate if changes were made. The images or other third party material in this article are included in the article's Creative Commons license, unless indicated otherwise in a credit line to the material. If material is not included in the article's Creative Commons license and your intended use is not permitted by statutory regulation or exceeds the permitted use, you will need to obtain permission directly from the copyright holder. To view a copy of this license, visit http://creativecommons.org/licenses/by/4.0/.

(C) The Author(s) 2019 